\title{
On the Etiological Relevance of Escherichia coli and Staphylococcus aureus in Superficial and Deep Infections - A Hypothesis-Forming, Retrospective Assessment
}

\author{
Hagen Frickmann ${ }^{1,2 *}$, Andreas Hahn², Stefan Berlec ${ }^{2}$, Johannes Ulrich², Moritz Jansson², \\ Norbert Georg Schwarz ${ }^{3}$, Philipp Warnke ${ }^{2}$ and Andreas Podbielski ${ }^{2}$ \\ ${ }^{1}$ Department of Microbiology and Hospital Hygiene, Bundeswehr Hospital Hamburg, Hamburg, Germany \\ ${ }^{2}$ Institute for Medical Microbiology, Virology, and Hygiene, University Medicine Rostock, Rostock, Germany \\ ${ }^{3}$ Infectious Disease Epidemiology, Bernhard Nocht Institute for Tropical Medicine Hamburg, Hamburg, Germany
}

Received: 26 Aug 2019; accepted: 08 Sep 2019

\begin{abstract}
Introduction: Escherichia coli and Staphylococcus aureus are important causes of severe diseases like blood stream infections. This study comparatively assessed potential differences in their impact on disease severity in local and systemic infections.

Methods: Over a 5-year interval, patients in whom either E. coli or S. aureus was detected in superficial or primary sterile compartments were assessed for the primary endpoint death during hospital stay and the secondary endpoints duration of hospital stay and infectious disease as the main diagnosis.

Results: Significance was achieved for the impacts as follows: Superficial infection with S. aureus was associated with an odds ratio of 0.27 regarding the risk of death and of 1.42 regarding infectious disease as main diagnosis. Superficial infection with $E$. coli was associated with a reduced duration of hospital stay by -2.46 days and a reduced odds ratio of infectious diseases as main diagnosis of 0.04 . The hospital stay of patients with $E$. coli was increased due to third-generation cephalosporin and ciprofloxacin resistance, and in the case of patients with $S$. aureus due to tetracycline and fusidic acid resistance.

Conclusions: Reduced disease severity of superficial infections due to both E. coli and S. aureus and resistancedriven prolonged stays in hospital were confirmed, while other outcome parameters were comparable.

Keywords: etiological relevance, infection, bacterium, virulence, resistance
\end{abstract}

\section{Introduction}

Staphylococcus aureus and Escherichia coli frequently cause superficial and systemic infections [1-10]. Further, both species are among the most frequent causes of bacteremia and sepsis in Western industrialized countries [11-16], with observed proportions of $16.3 \%$ to $21.6 \%$ for $S$. aureus and $5.6 \%$ to $24.2 \%$ for $E$. coli among all causes of sepsis, associated with considerable morbidity and mortality $[17,18]$. Prolonged antibiotic therapy is recommended for $S$. aureus-associated bacteremia due to high risk of secondary foci of infection and particularly high mortality $[19,20]$, both for methicillin-susceptible and for methicillin-resistant strains [17]. Along with systemic infections, both species can play a role in superficial infections such as wound infections [21-23] or in urinary tract infections [24, 25].

While the etiological relevance of both of these facultatively pathogenic species under the conditions described can be considered as well documented, the pathogenic potential of individual strains depends on the presence or absence of pathogenic factors and toxins [26, 27] and may vary. The pathogenic factors of $E$. coli comprise adhesins (fimbrial as well as afimbrial ones and outer membrane proteins), curli, flagella, fimbriae, invasins, iron acquisition factors (siderophores), lipo-

\footnotetext{
*Author for correspondence: Department of Microbiology and Hospital Infection, Bundeswehr Hospital Hamburg, Bernhard Nocht Str. 74, 20359 Hamburg, Germany; E-mail: frickmann@bnitm.de; Tel: 0049-40-6947-28743; Fax: 004040-6947-28709.
}

polysaccharides, pili, polysaccharide capsules, secreted serine proteases, and metalloproteases, and toxins like oligopeptides, $\mathrm{AB}$ (alpha and beta subunit)-toxins, and RTX (repeats in toxin) pore-forming toxins $[25,26,28-32]$. For $S$. aureus, agglutinins, coagulases and staphylokinases, exoenzymes like nucleases and proteases, secreted toxins such as host protease modulators, pore-forming toxins, and superantigens, as well as the ability of forming biofilms due to cell surface-associated proteins, are among the described pathogenic factors [33-41]. As recently discussed elsewhere [42, 43], resistance against bactericidal first-line drugs like beta-lactam antibiotics, caused by enzymes like, e.g., extended spectrum beta-lactamases (ESBL) or carbapenems for E. coli, as well as by penicillinbinding proteins for methicillin-resistant $S$. aureus (MRSA), can become a severe problem for medical care.

However, previous studies have suggested a trade-off between resistance and pathogenicity [44, 45], assuming that expressing resistance determinants may cost additional energy and could thus decrease the fitness and competitiveness of the strain. Highly varying resistance rates have been observed in $S$. aureus and $E$. coli strains in previous assessments [2, 6, 7].

This study was conducted to provide hypotheses to be proven by future prospective studies, focusing on two questions. Firstly, we have performed a comparative head-to-head assessment of various systemic or superficial infections exclusively associated either with $S$. aureus or with $E$. coli to get hints on potentially differing disease severity as measured by the outcome parameters, namely, death during hospital stay, duration of hospital stay, and infectious disease as the main

This is an open-access article distributed under the terms of the Creative Commons Attribution-NonCommercial 4.0 International License (https://creativecommons.org/licenses/by-nc/4.0/), which permits unrestricted use, distribution, and reproduction in any medium for non-commercial purposes, provided the original author and source are credited, a link to the CC License is provided, and changes - if any - are indicated. 
diagnosis. Secondly, associations of resistance with the clinical course of documented infections have been assessed to discern hints supporting the above-mentioned fitness cost hypothesis.

\section{Patients and Methods}

2.1. Study Design. The assessment was conducted as a single-center retrospective observational study over 5 years at a German university hospital. Inclusion criteria will be discussed in detail later under the respective heading. Data were obtained from a laboratory information system (LIS) of the DIN EN ISO 15189-accredited Institute for Medical Microbiology, Virology, and Hygiene of the University Medicine Rostock, Germany. In detail, cases were identified by screening for the search terms: Staphylococcus aureus and Escherichia coli.

For the identification of the bacterial isolates assessed in this study, VITEK 2 identification cards (bioMérieux, Marcyl'Étoile, France) or matrix-assisted laser desorption/ionization time-of-flight mass spectrometry (MALDI-TOF-MS) with a Shimadzu/Kratos "AXIMA Assurance" MALDI-TOF mass spectrometer (Shimadzu Germany Ltd., Duisburg, Germany) were used. As described by the manufacturer (bioMérieux), alpha-cyano-4-hydroxycinnamic acid preparation was carried out for all strains prior to MALDI-TOF assessment. The spectral fingerprints obtained were interpreted using the Vitek MSID IVD-mode database version 3.2.0.-6. (bioMérieux). The equivalence of these methods regarding their diagnostic reliability has repeatedly been confirmed in the literature [46-49]. Antibiotic resistance was analyzed applying Clinical and Laboratory Standards Institute ((CLSI), CLSI M100-S17/M2-A9, M7-A7 January 2007; CLSI M100-S19, M2-A8, M7-A8 January 2009) and European Committee on Antimicrobial Susceptibility Testing ((EUCAST), Clinical Break Point Version 2 January 2012 and Version 3 January 2013) clinical breakpoints using the appropriate VITEK 2 AST cards in the course of the study. No adjustment for breakpoint changes was performed. Assessed antimicrobials comprised penicillins (ampicillin, ampicillin-sulbactam, oxacillin, and piperacillintazobactam), cephalosporins (3rd and 4th generation cephalosporins, as well as cefuroxime and cefoxitin), the carbapenem imipenem, fluoroquinolones (norfloxacin, ciprofloxacin, levofloxacin, and moxifloxacin), aminoglycosides (gentamicin and tobramycin), glycopeptides (vancomycin and teicoplanin), the macrolide erythromycin, the lincosamide clindamycin, the streptogramin quinupristin, tetracycline, the glycylcycline tigecycline, the drug combination co-trimoxazole, fosfomycin, fusidic acid, rifampicin, the oxazolidinone linezolid, the lipopeptide daptomycin, and mupirocin.

After the removal of copy strains, except for the first isolate of each case, inpatient cases were assessed anonymously. Each patient was counted only once. Data were anonymously extracted from the patients' case files and collected in Microsoft Excel worksheets for statistical analysis.

2.2. Outcome Parameters. Primary and secondary outcome parameters were defined, with death during hospital stay as the primary outcome parameter and the duration of hospital stay, as well as presence or absence of an infectious disease as main diagnosis (localized or systemic infections) versus a non-infectious disease-related main diagnosis (from the fields of cardiovascular diseases, endocrinology, gastroenterology, neurology, orthopedics and trauma surgery, pneumology, rheumatology and tumors, urology, and others) as secondary outcome parameters.

The outcome parameters were assessed for patients with infections due to either $S$. aureus or E. coli with additional focus on superficial and systemic infections. Superficial infections assessed comprised skin and urinary tract infections, while lower respiratory tract infections, bacteremia, and infections of primary sterile compartments were defined as systemic infections.

Association of antibiotic resistance with the assessed outcome-parameter was also investigated.

2.3. Factors Potentially Affecting the Outcome. In addition to the outcome parameters described above, a number of factors were documented to assess potential effects on the outcome parameters. These variables comprised continuous parameters, such as age, and also noncontinuous parameters, such as gender; isolation site of the strain; non-surgical ward vs. surgical and intensive care ward; main diagnoses from the fields of cardiovascular disease, endocrinology/metabolic disorders, gastroenterology, local infections or systemic infections, neurology, orthopedics/traumatology, pulmonology, rheumatology, neoplasia, urology and others; peak values of leukocytes; procalcitonin (PCT) and C-reactive protein (CRP); and the presence or absence of antibiotic treatment at the time of hospital admission. Leukocytes, CRP, and PCT were semiquantitatively categorized as shown in Table 1. Sample materials in which $S$. aureus or $E$. coli were identified were grouped as abscess materials, ascites, aspirates, biopsies and invasive foreign material, blood cultures, bronchial lavage, respiratory secretions, wound swabs, and urine. Antibiotic susceptibility or resistance as defined by EUCAST was recorded for comparison within the species.

The distribution of both the outcome parameters and potentially confounding variables is presented in Table 2 for both S. aureus and E. coli.

2.4. Inclusion and Exclusion Criteria. Patients were included if either $S$. aureus or $E$. coli was identified in the microbiological laboratory in any clinical sample material and if clinical information from the case files was available.

Incompleteness of the assessable dataset alone was not considered as exclusion criterion, although it led to a reduction in the number of interpretable cases.

2.5. Statistical Assessment. Statistical assessment was done using STATA 15.1 (StataCorp, USA) with an exploratory aim. Binary logistic regression was used for the binary endpoint parameters, namely, death and infectious disease as main diagnosis. Linear regression was used for the endpoint parameter duration of hospital stay. All models were implemented as backward selection models with a significance level of 0.1 to exclude parameters from the model. Modeling was performed for the whole dataset (global modeling), as well as for patients with either $S$. aureus or E. coli (local modeling). Comparisons between disjoint subpopulations generally refer to their complement. As there have been no defined references in this study, for practical reasons, the category with the lowest score or the first

Table 1. Algorithm of semi-quantification of leukocyte counts as well as CRP and PCT concentrations

\begin{tabular}{|c|c|c|c|c|c|c|}
\hline Parameter & Unit & Reference value & Category 1 & Category 2 & Category 3 & Category 4 \\
\hline Leukocyte count & $10^{9} / \mathrm{L}$ & $4-9$ & Reduced $(<4)$ & Normal (4-9) & Increased $(>9)$ & \\
\hline CRP & $\mathrm{mg} / \mathrm{L}$ & $<5$ & Normal $(<5)$ & $\begin{array}{l}\text { Slightly increased } \\
\qquad 5-50)\end{array}$ & & $\begin{array}{l}\text { Severely increased } \\
\qquad(>100)\end{array}$ \\
\hline PCT & $\mathrm{ng} / \mathrm{L}$ & $<0.06$ & Normal $(<0.06)$ & $\begin{array}{l}\text { Slightly increased } \\
(>0.06-10)\end{array}$ & $\begin{array}{l}\text { Moderately increased } \\
\qquad>10-100)\end{array}$ & $\begin{array}{l}\text { Severely increased } \\
\qquad(>100)\end{array}$ \\
\hline
\end{tabular}


Table 2. Overview on the distribution of individually assessed parameters in patients with $S$. aureus or E. coli infections

\begin{tabular}{|c|c|c|c|}
\hline Parameter & $\begin{array}{l}S . \text { aureus } \\
n=1040\end{array}$ & $\begin{array}{c}E . \text { coli } \\
n=975\end{array}$ & $\begin{array}{c}\text { Overall } \\
n=2015\end{array}$ \\
\hline Death $n(\%)$ & $128(12.4 \%)$ & $91(9.3 \%)$ & $219(10.9 \%)$ \\
\hline \multicolumn{4}{|l|}{ Gender } \\
\hline Male & $648(62.3 \%)$ & $423(43.4 \%)$ & $1071(53.2 \%)$ \\
\hline Female & $392(37.7 \%)$ & $552(56.6 \%)$ & $944(46.9 \%)$ \\
\hline \multicolumn{4}{|l|}{ Patient group } \\
\hline Systemic infection & $405(38.9 \%)$ & $353(36.2 \%)$ & $758(37.6 \%)$ \\
\hline Superficial infection & $557(53.6 \%)$ & $482(49.4 \%)$ & $1039(51.6 \%)$ \\
\hline $\begin{array}{l}\text { Combined superficial } \\
\text { and systemic infection }\end{array}$ & $78(5.5 \%)$ & $140(14.4 \%)$ & $218(10.8 \%)$ \\
\hline \multicolumn{4}{|l|}{ Sample materials } \\
\hline Ascites & $3(0.3 \%)$ & $10(1.0 \%)$ & $13(0.7 \%)$ \\
\hline $\begin{array}{l}\text { Biopsies and invasive } \\
\text { foreign material }\end{array}$ & $47(4.5 \%)$ & $428(43.9 \%)$ & $475(23.6 \%)$ \\
\hline Blood cultures & $234(22.5 \%)$ & $245(25.1 \%)$ & $479(23.8 \%)$ \\
\hline Bronchial lavage & $17(1.6 \%)$ & $19(2.0 \%)$ & $36(1.8 \%)$ \\
\hline Respiratory secretions & $127(12.2 \%)$ & $37(3.8 \%)$ & $164(8.1 \%)$ \\
\hline Aspirates & $14(1.4 \%)$ & $35(3.6 \%)$ & $49(2.4 \%)$ \\
\hline Abscess materials & $70(6.7 \%)$ & $73(7.5 \%)$ & $143(7.1 \%)$ \\
\hline Wound swabs & $501(48.2 \%)$ & $27(2.8 \%)$ & $528(26.2 \%)$ \\
\hline Urine & $27(2.6 \%)$ & $101(10.4 \%)$ & $128(6.4 \%)$ \\
\hline \multicolumn{4}{|l|}{ Main diagnoses } \\
\hline $\begin{array}{l}\text { Endocrinology/metabolic } \\
\text { disorders }\end{array}$ & $16(1.5 \%)$ & $4(0.4 \%)$ & $20(1.0 \%)$ \\
\hline Gastroenterology & $56(5.4 \%)$ & $175(17.8 \%)$ & $231(11.5 \%)$ \\
\hline Cardiovascular disease & $99(9.5 \%)$ & $82(8.4 \%)$ & $181(9.0 \%)$ \\
\hline Local infection & $333(32.0 \%)$ & $35(3.6 \%)$ & $368(18.3 \%)$ \\
\hline Systemic infection & $103(9.9 \%)$ & $183(18.8 \%)$ & $286(14.2 \%)$ \\
\hline $\begin{array}{l}\text { Rheumatology and } \\
\text { neoplasia }\end{array}$ & $108(10.4 \%)$ & $131(13.4 \%)$ & $239(11.7 \%)$ \\
\hline Neurological disorder & $116(11.2 \%)$ & $24(2.5 \%)$ & $140(7.0 \%)$ \\
\hline Orthopedics/Traumatology & $79(7.6 \%)$ & $24(2.4 \%)$ & $103(5.1 \%)$ \\
\hline Pulmonary disease & $60(5.8 \%)$ & $80(8.2 \%)$ & $140(7.0 \%)$ \\
\hline Other diseases & $37(3.6 \%)$ & $50(5.1 \%)$ & $87(4.3 \%)$ \\
\hline Urologic disease & $33(3.2 \%)$ & $187(19.2 \%)$ & $220(10.9 \%)$ \\
\hline \multicolumn{4}{|l|}{ Ward } \\
\hline Surgical & $422(40.6 \%)$ & $394(40.4 \%)$ & $816(40.5 \%)$ \\
\hline Medical & $618(59.4 \%)$ & $581(59.6 \%)$ & $1199(59.5 \%)$ \\
\hline \multicolumn{4}{|l|}{$\begin{array}{l}\text { Previous antibiotic } \\
\text { therapy }\end{array}$} \\
\hline Not documented & $594(57.1 \%)$ & $665(68.2 \%)$ & $1259(62.5 \%)$ \\
\hline Documented & $446(42.9 \%)$ & $310(31.8 \%)$ & $756(37.5 \%)$ \\
\hline \multicolumn{4}{|l|}{ Ampicillin } \\
\hline Susceptible & $40(37.7 \%)$ & $462(47.7 \%)$ & $502(46.7 \%)$ \\
\hline Resistant & $66(62.3 \%)$ & $507(52.3 \%)$ & $573(53.3 \%)$ \\
\hline \multicolumn{4}{|l|}{ Levofloxacin } \\
\hline Susceptible & $841(85.8 \%)$ & n.a. & $841(85.8 \%)$ \\
\hline Resistant & $139(14.2 \%)$ & n.a. & $139(14.2 \%)$ \\
\hline \multicolumn{4}{|l|}{ Norfloxacin } \\
\hline Susceptible & $220(85.9 \%)$ & n.a. & $220(85.9 \%)$ \\
\hline Resistant & $36(14.1 \%)$ & n.a. & $36(14.1 \%)$ \\
\hline \multicolumn{4}{|l|}{ Ciprofloxacin } \\
\hline Susceptible & $221(86.0 \%)$ & $728(75.2 \%)$ & $949(77.5 \%)$ \\
\hline Resistant & $36(14.0 \%)$ & $240(24.8 \%)$ & $276(22.5 \%)$ \\
\hline \multicolumn{4}{|l|}{ Moxifloxacin } \\
\hline Susceptible & $860(87.8 \%)$ & n.a. & $860(87.8 \%)$ \\
\hline Resistant & $119(12.2 \%)$ & n.a. & $119(12.2 \%)$ \\
\hline \multicolumn{4}{|l|}{ Erythromycin } \\
\hline Susceptible & $880(89.8 \%)$ & n.a. & $880(89.8 \%)$ \\
\hline Resistant & $100(10.2 \%)$ & n.a. & $100(10.2 \%)$ \\
\hline Clindamycin & & & \\
\hline Susceptible & $885(90.3 \%)$ & n.a. & $885(90.3 \%)$ \\
\hline Resistant & $95(9.7 \%)$ & n.a. & $95(9.7 \%)$ \\
\hline Quinupristin & & & \\
\hline Susceptible & $256(100 \%)$ & n.a. & $256(100 \%)$ \\
\hline Resistant & 0 & n.a. & 0 \\
\hline Gentamicin & & & \\
\hline Susceptible & $957(97.8 \%)$ & $915(94.3 \%)$ & $1872(96.1 \%)$ \\
\hline Resistant & $22(2.3 \%)$ & $55(5.7 \%)$ & $77(4.0 \%)$ \\
\hline Tetracycline & & & \\
\hline Susceptible & 905 (92.4\%) & $390(61.2 \%)$ & $1295(80.1 \%)$ \\
\hline Resistant & $74(7.6 \%)$ & $247(38.8 \%)$ & $321(19.9 \%)$ \\
\hline Tobramycin & & & \\
\hline Susceptible & $822(97.4 \%)$ & n.a. & $822(97.4 \%)$ \\
\hline Resistant & $22(2.6 \%)$ & n.a. & $22(2.6 \%)$ \\
\hline Tigecycline & & & \\
\hline
\end{tabular}

Table 2. (contd.)

\begin{tabular}{|c|c|c|c|}
\hline Parameter & $\begin{array}{l}\text { S. aureus } \\
n=1040\end{array}$ & $\begin{array}{c}\text { E. coli } \\
n=975\end{array}$ & $\begin{array}{c}\text { Overall } \\
n=2015\end{array}$ \\
\hline Susceptible & $722(100 \%)$ & $426(100 \%)$ & $1148(100 \%)$ \\
\hline Resistant & 0 & 0 & 0 \\
\hline \multicolumn{4}{|l|}{ Co-trimoxazole } \\
\hline Susceptible & $972(99.3 \%)$ & $686(70.4 \%)$ & $1658(84.9 \%)$ \\
\hline Resistant & $7(0.7 \%)$ & $289(29.6 \%)$ & $296(15.2 \%)$ \\
\hline \multicolumn{4}{|l|}{ Fosfomycin } \\
\hline Susceptible & $972(99.4 \%)$ & $334(99.4 \%)$ & $1306(99.4 \%)$ \\
\hline Resistant & $6(0.6 \%)$ & $2(0.6 \%)$ & $8(0.6 \%)$ \\
\hline \multicolumn{4}{|l|}{ Fusidic acid } \\
\hline Susceptible & $950(97.5 \%)$ & n.a. & $950(97.5 \%)$ \\
\hline Resistant & $24(2.5 \%)$ & n.a. & $24(2.5 \%)$ \\
\hline \multicolumn{4}{|l|}{ Rifampicin } \\
\hline Susceptible & $976(99.7 \%)$ & n.a. & $976(99.7 \%)$ \\
\hline Resistant & $3(0.3 \%)$ & n.a. & $3(0.3 \%)$ \\
\hline \multicolumn{4}{|l|}{ Oxacillin } \\
\hline Susceptible & $1035(100 \%)$ & n.a. & $1035(100 \%)$ \\
\hline Resistant & 0 & n.a. & 0 \\
\hline \multicolumn{4}{|l|}{ Ampicillin-Sulbactam } \\
\hline Susceptible & $948(100 \%)$ & $580(65.2 \%)$ & $1528(83.1 \%)$ \\
\hline Resistant & 0 & $310(84.8 \%)$ & $310(16.9 \%)$ \\
\hline \multicolumn{4}{|l|}{ Cefoxitin } \\
\hline Susceptible & $964(100 \%)$ & n.a. & $964(100 \%)$ \\
\hline Resistant & 0 & n.a. & 0 \\
\hline \multicolumn{4}{|l|}{ Cefuroxime } \\
\hline Susceptible & $948(100 \%)$ & n.a. & $948(100 \%)$ \\
\hline Resistant & 0 & n.a. & 0 \\
\hline \multicolumn{4}{|l|}{ Imipenem } \\
\hline Susceptible & $948(100 \%)$ & $974(100 \%)$ & $1922(100 \%)$ \\
\hline Resistant & 0 & 0 & 0 \\
\hline \multicolumn{4}{|l|}{ Teicoplanin } \\
\hline Susceptible & $978(100 \%)$ & n.a. & $978(100 \%)$ \\
\hline Resistant & 0 & n.a. & 0 \\
\hline \multicolumn{4}{|l|}{ Vancomycin } \\
\hline Susceptible & $978(100 \%)$ & n.a. & $978(100 \%)$ \\
\hline Resistant & 0 & n.a. & 0 \\
\hline Linezolid & & & \\
\hline Susceptible & $980(100 \%)$ & n.a. & $980(100 \%)$ \\
\hline Resistant & 0 & n.a. & 0 \\
\hline Daptomycin & & & \\
\hline Susceptible & $137(99.3 \%)$ & n.a. & $137(99.3 \%)$ \\
\hline Resistant & $1(0.7 \%)$ & n.a. & $1(0.7 \%)$ \\
\hline Mupirocin & & & \\
\hline Susceptible & $963(99.8 \%)$ & n.a. & $963(99.8 \%)$ \\
\hline Resistant & $2(0.2 \%)$ & n.a. & $2(0.2 \%)$ \\
\hline $\begin{array}{l}\text { Third-generation } \\
\text { cephalosporins }\end{array}$ & & & \\
\hline Susceptible & n.a. & $835(88.6 \%)$ & $835(88.6 \%)$ \\
\hline Resistant & n.a. & $108(11.5 \%)$ & $108(11.5 \%)$ \\
\hline $\begin{array}{l}\text { Fourth-generation } \\
\text { cephalosporins }\end{array}$ & & & \\
\hline Susceptible & n.a. & $901(92.6 \%)$ & $901(92.6 \%)$ \\
\hline Resistant & n.a. & $72(7.4 \%)$ & $72(7.4 \%)$ \\
\hline Piperacillin-Tazobactam & & & \\
\hline Susceptible & n.a. & $747(85.4 \%)$ & $747(85.4 \%)$ \\
\hline Resistant & n.a. & $128(14.6 \%)$ & $128(14.6 \%)$ \\
\hline $\begin{array}{l}\text { Days in hospital } \\
\text { (available for }(n) \text { ) }\end{array}$ & 1440 & 974 & 2014 \\
\hline Mean (SD) & $19.6(19.2)$ & $15.3(16.3)$ & $17.6(18.0)$ \\
\hline Median & 14.0 & 10.0 & 12.0 \\
\hline $\begin{array}{l}\text { Days to sample } \\
\text { acquisition } \\
\text { (available for }(n) \text { ) }\end{array}$ & 1040 & 975 & 2015 \\
\hline Mean (SD) & $4.7(10.4)$ & $3.8(7.5)$ & $4.3(9.1)$ \\
\hline Median & 1.0 & 1.0 & 1.0 \\
\hline Age (available for $(n)$ ) & 1040 & 975 & 2015 \\
\hline Mean (SD) & $58.3(22.0)$ & $69.3(15.1)$ & $63.6(19.7)$ \\
\hline Median & 63.0 & 72.0 & 69.0 \\
\hline CRP (available for $(n)$ ) & 1009 & 899 & 1908 \\
\hline Mean (SD) & $1.9(1.1)$ & $2.1(1.0)$ & $2.0(1.0)$ \\
\hline Median & 2.0 & 2.0 & 2.0 \\
\hline PCT (available for $(n)$ ) & 242 & 157 & 399 \\
\hline Mean (SD) & $1.2(0.5)$ & $1.3(0.2)$ & $1.3(0.5)$ \\
\hline Median & 1.0 & 1.0 & 1.0 \\
\hline $\begin{array}{l}\text { Leukocytes } \\
\text { (available for }(n) \text { ) }\end{array}$ & 998 & 975 & 1973 \\
\hline Mean (SD) & $2.5(0.6)$ & $2.5(0.6)$ & $2.5(0.6)$ \\
\hline Median & 3.0 & 3.0 & 3.0 \\
\hline
\end{tabular}


Table 3. Explorative binary logistic regression model with backward selection for the global model with patients with $S$. aureus and/or E. coli infections. Association with the outcome parameter death

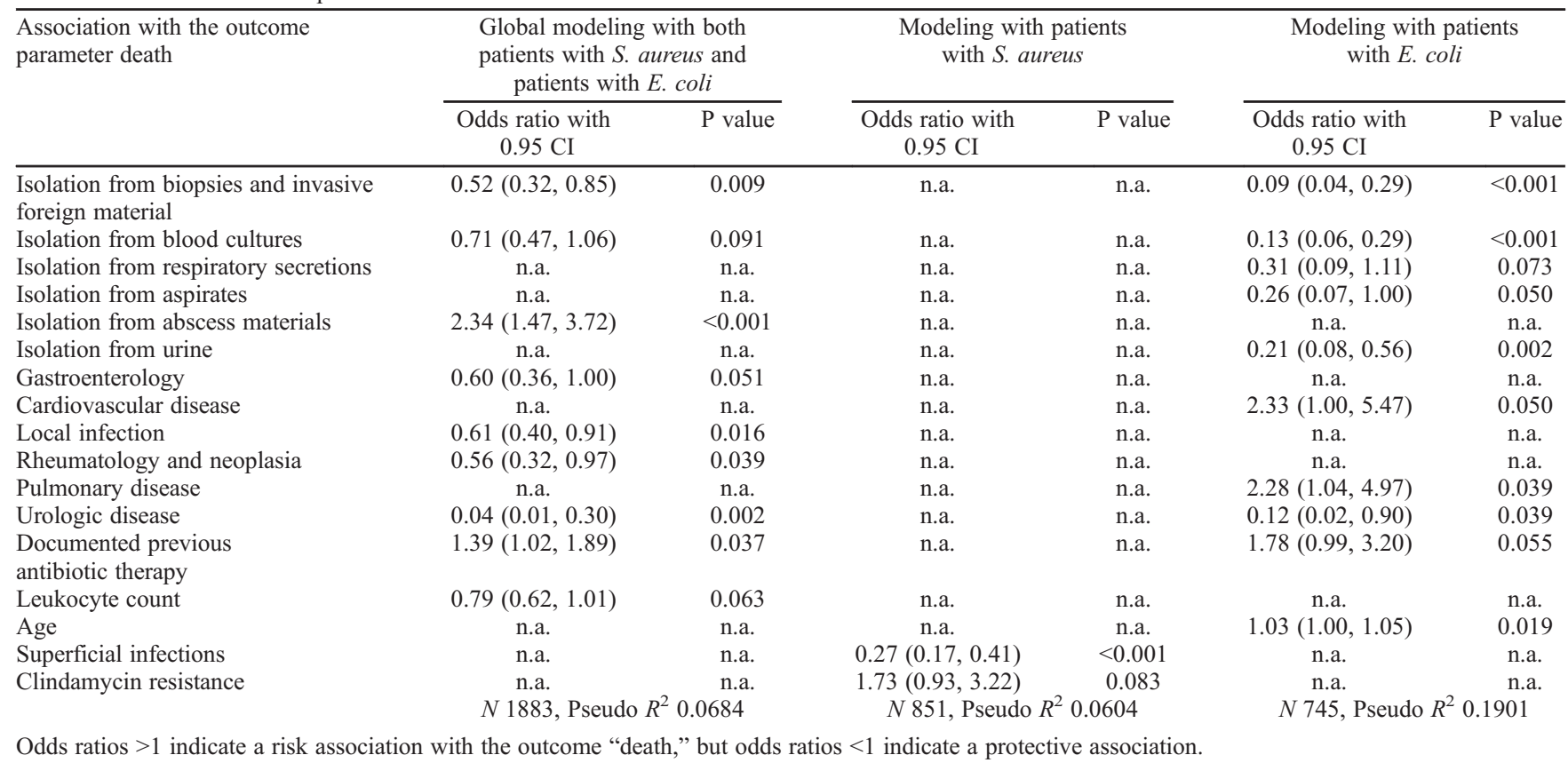

Table 4. Explorative linear regression model with backward selection for the global model with patients with $S$. aureus and/or E. coli infections. Association with the outcome parameter duration of hospital stay

\begin{tabular}{|c|c|c|c|c|c|c|}
\hline \multirow[t]{2}{*}{$\begin{array}{l}\text { Association with the outcome parameter } \\
\text { duration of hospital stay }\end{array}$} & \multicolumn{2}{|c|}{$\begin{array}{l}\text { Global modeling with both } \\
\text { patients with } S \text {. aureus and } \\
\text { patients with } E \text {. coli }\end{array}$} & \multicolumn{2}{|c|}{$\begin{array}{l}\text { Modeling with patients } \\
\quad \text { with } S \text {. aureus }\end{array}$} & \multicolumn{2}{|c|}{$\begin{array}{l}\text { Modeling with patients } \\
\text { with } E \text {. coli }\end{array}$} \\
\hline & $\begin{array}{l}\text { Odds ratio with } \\
0.95 \mathrm{CI}\end{array}$ & $P$ value & $\begin{array}{l}\text { Odds ratio with } \\
0.95 \mathrm{CI}\end{array}$ & $P$ value & $\begin{array}{l}\text { Odds ratio with } \\
0.95 \mathrm{CI}\end{array}$ & $P$ value \\
\hline Biopsies and invasive foreign material & $-1.36(-2.23,-0.49)$ & 0.002 & n.a. & n.a. & $-2.00(-3.14,-0.88)$ & 0.001 \\
\hline Blood cultures & n.a. & n.a. & $1.40(0.08,2.71)$ & 0.037 & $-3.15(-4.49,-1.81)$ & $<0.001$ \\
\hline Bronchial lavage & n.a. & n.a. & n.a. & n.a. & $-5.51(-8.45,-2.56)$ & $<0.001$ \\
\hline Respiratory secretions & n.a. & n.a. & $2.16(0.52,3.80)$ & 0.010 & $-2.89(-5.48,-0.29)$ & 0.029 \\
\hline Aspirates & n.a. & n.a. & n.a. & n.a. & $-2.55(-4.66,-0.44)$ & 0.018 \\
\hline Wound swabs & $-1.16(-1.98,-0.34)$ & 0.005 & n.a. & n.a. & n.a. & n.a. \\
\hline Gastroenterology & $3.18(2.11,4.25)$ & $<0.001$ & $5.18(2.84,7.52)$ & $<0.001$ & $2.57(1.36,3.79)$ & $<0.001$ \\
\hline Cardiovascular disease & $1.68(0.50,2.85)$ & 0.005 & n.a. & n.a. & $2.26(0.70,3.81)$ & 0.005 \\
\hline Rheumatology and neoplasia & $2.90(1.79,4.01)$ & $<0.001$ & $2.98(1.01,4.06)$ & 0.003 & $2.86(1.54,4.17)$ & $<0.001$ \\
\hline Pulmonary disease & $2.01(0.72,3.30)$ & 0.002 & n.a. & n.a. & $2.83(1.27,4.38)$ & $<0.001$ \\
\hline Other diseases & $2.79(1.14,4.43)$ & 0.001 & $3.15(0.19,6.19)$ & 0.037 & $2.39(0.45,4.32)$ & 0.016 \\
\hline Urologic disease & $1.99(0.85,3.13)$ & 0.001 & n.a. & n.a. & $2.18(0.99,3.38)$ & $<0.001$ \\
\hline Previous antibiotic therapy & $1.46(0.79,2.13)$ & $<0.001$ & $2.10(1.01,3.20)$ & $<0.001$ & n.a. & n.a. \\
\hline Age & $-0.036(-0.05,-0.02)$ & $<0.001$ & $-0.04(-0.06,-0.01)$ & 0.002 & n.a. & n.a. \\
\hline Third-generation cephalosporin resistance & n.a. & n.a. & n.a. & n.a. & $2.59(1.38,3.81)$ & $<0.001$ \\
\hline Tetracycline resistance & n.a. & n.a. & $2.22(0.25,4.18)$ & 0.027 & n.a. & n.a. \\
\hline Fusidic acid resistance & n.a. & n.a. & $5.67(2.26,9.08)$ & 0.001 & n.a. & n.a. \\
\hline Ciprofloxacin resistance & n.a. & n.a. & n.a. & n.a. & $0.81(-0.15,1.76)$ & 0.097 \\
\hline Superficial infection & $\begin{array}{c}\text { n.a. } \\
N 1883 \text {, adjusted } R^{2}\end{array}$ & $\begin{array}{l}\text { n.a. } \\
3987\end{array}$ & $\begin{array}{c}\text { n.a. } \\
N 918, \text { adjusted } R\end{array}$ & $\begin{array}{l}\text { n.a. } \\
3863\end{array}$ & $\begin{array}{r}-2.46(-3.71,-1.21) \\
N 774, \text { adjusted } R^{2}\end{array}$ & $\begin{array}{l}<0.001 \\
.5340\end{array}$ \\
\hline
\end{tabular}

Coefficients $>0$ indicate a prolonging association with the endpoint days to discharge (parameter extends on average by the shown number of days), coefficients $<0$ indicate a shortening association (parameter reduces on average by the shown number of days).

appearance in each group has been used as a reference for the calculations. Parameters with insufficient numbers for regression analysis were excluded from the modeling. For the endpoint-parameter infectious disease as a main diagnosis, the parameters of main diagnoses were not included into the model due to lack of independence.

2.6. Ethics. Ethical clearance for the assessment was obtained from the Ethics Committee of the University Medicine Rostock (Registration number A 2014-0054). The study was performed in accordance with the ethical standards laid down in the 1964 Declaration of Helsinki and its later amendments. Do to the retrospective design of the study, the assessment was allowed by the ethics committee in an anonymous way without consent to participate.

\section{Results}

3.1. Study Population. After removal of copy strains, the study population comprised 2015 cases: 1040 isolations of $S$. aureus and 975 isolations of $E$. coli. These isolations were associated with systemic infections in 758 cases, with superficial infections in 1039 cases, and with combined systemic and superficial infections in 218 cases. 
Table 5. Explorative binary logistic regression model with backward selection for the global model with patients with $S$. aureus and/or $E$. coli infections. Association with the outcome parameter infectious disease as main diagnosis

\begin{tabular}{|c|c|c|c|c|c|c|}
\hline \multirow[t]{2}{*}{$\begin{array}{l}\text { Association with the outcome } \\
\text { parameter infectious disease } \\
\text { as main diagnosis }\end{array}$} & \multicolumn{2}{|c|}{$\begin{array}{l}\text { Global modeling with both } \\
\text { patients with } S \text {. aureus and } \\
\text { patients with } E \text {. coli }\end{array}$} & \multicolumn{2}{|c|}{$\begin{array}{l}\text { Modeling with patients } \\
\text { with } S \text {. aureus }\end{array}$} & \multicolumn{2}{|c|}{$\begin{array}{l}\text { Modeling with patients } \\
\text { with } E \text {. coli }\end{array}$} \\
\hline & $\begin{array}{c}\text { Odds ratio with } \\
0.95 \mathrm{CI}\end{array}$ & $\overline{P \text { value }}$ & $\begin{array}{c}\text { Odds ratio with } \\
0.95 \mathrm{CI}\end{array}$ & $\overline{\mathrm{P} \text { value }}$ & $\begin{array}{c}\text { Odds ratio with } \\
0.95 \mathrm{CI}\end{array}$ & $P$ value \\
\hline $\begin{array}{l}\text { Biopsies and invasive } \\
\text { foreign material }\end{array}$ & n.a. & n.a. & n.a. & n.a. & $4.26(1.78,10.19)$ & 0.001 \\
\hline Blood cultures & $5.53(4.15,7.38)$ & $<0.001$ & n.a. & n.a. & $5.69(2.78,11.68)$ & $<0.001$ \\
\hline Bronchial lavage & n.a. & n.a. & $0.23(0.05,1.07)$ & 0.061 & n.a. & n.a. \\
\hline Respiratory secretions & n.a. & n.a. & $0.20(0.11,0.37)$ & $<0.001$ & n.a. & n.a. \\
\hline Aspirates & $5.40(2.92,10.01)$ & $<0.001$ & n.a. & n.a. & $14.19(5.24,38.40)$ & $<0.001$ \\
\hline Abscess materials & $5.52(3.61,8.46)$ & $<0.001$ & $4.88(2.58,9.25)$ & $<0.001$ & n.a. & n.a. \\
\hline Wound swabs & $8.08(6.06,10.77)$ & $<0.001$ & $1.83(1.33,2.51)$ & $<0.001$ & $651.55(157.87,2689.04)$ & $<0.001$ \\
\hline Urine & n.a. & n.a. & $0.13(0.03,0.60)$ & 0.008 & $18.99(6.41,56.22)$ & $<0.001$ \\
\hline Age & $0.99(0.98,0.99)$ & $<0.001$ & $0.99(0.98,0.99)$ & 0.001 & $1.01(1.00,1.03)$ & 0.083 \\
\hline Gender & $1.25(1.00,1.54)$ & 0.045 & n.a. & n.a. & n.a. & n.a. \\
\hline Leukocyte count & $1.47(1.22,1.75)$ & $<0.001$ & $1.35(1.06,1.73)$ & 0.016 & $1.71(1.22,2.41)$ & 0.002 \\
\hline CRP & n.a. & n.a. & $0.86(0.74,0.99)$ & 0.034 & $1.43(1.12,1.81)$ & 0.004 \\
\hline \multirow[t]{2}{*}{$\begin{array}{l}\text { Surgical and intensive } \\
\text { care wards }\end{array}$} & n.a. & n.a. & n.a. & n.a. & $0.18(0.11,0.30)$ & $<0.001$ \\
\hline & \multicolumn{2}{|c|}{$N$ 1885, Pseudo $R^{2} 0.1385$} & \multicolumn{2}{|c|}{$N$ 986, Pseudo $R^{2} 0.1292$} & \multicolumn{2}{|c|}{$N$ 899, Pseudo $R^{2} 0.3473$} \\
\hline
\end{tabular}

Odds ratios $>1$ indicate a risk association with the outcome infectious disease as main diagnosis, whereas odds ratios $<1$ indicate a protective association.

3.2. Assessed Parameters. Detailed information on the distribution of assessed patient and strain characteristics is provided in Table 2. Thereby, parameters are presented as distributed by either species of the bacterial isolates $(S$. aureus or E. coli), as well as for the whole assessed population. Based on those data, global modeling for the whole dataset, as well as local modeling for patients with either $S$. aureus or $E$. coli with a focus on the study endpoints, was performed; the results are presented in the following sections.

3.3. Assessment of the Study Endpoints. Neither isolation of $S$. aureus nor that of $E$. coli was associated with any of the endpoint parameters in the global modeling. However, superficial infection with $S$. aureus was negatively associated with the primary outcome parameter death $(\mathrm{P}<0.001)$, but it was positively associated with infectious disease as the main diagnosis $(P=0.013)$. Superficial infection with $E$. coli, in contrast, was associated with shorter duration of hospital stay $(P<0.001)$ and negatively associated with infectious disease as main diagnosis $(P<0.001)$. Several variables potentially affected the primary and secondary outcome parameters as shown in Tables 3-5.

3.4. Associations between Resistance and Superficial or Systemic Infections. Enhanced resistance, i.e., resistance to 3 or more antibiotic substance classes, was not generally positively or negatively associated with invasive infections.

Resistance against tetracycline $(P=0.027)$ and fusidic acid $(P<0.001)$ in patients with $S$. aureus and against third-generation cephalosporins $(P<0.001)$ and ciprofloxacin $(P=0.097)$ in patients with $E$. coli was associated with increased duration of hospital stay. Also, there was a tendency for an increased risk of death in patients with clindamycinresistant $S$. aureus $(P=0.083)$ (Tables 3 and 4).

\section{Discussion}

The study was conducted to analyze any differences in the etiological relevance of $S$. aureus and E. coli over a study period of 5 years with inpatients at a German university hospital with superficial or systemic infections. The focus was on the primary endpoint death during hospital stay, and the two secondary endpoints, duration of hospital stay and a main diagnosis of infectious disease. Potential associations between resistance and severity of the infectious diseases were also assessed.
The results of the assessment differed for the different cases. First of all, neither $S$. aureus nor E. coli as individual species alone were associated with any of the outcome parameters. As expected, superficial infections with $S$. aureus were associated with reduced risk of death compared with systemic infections; most interestingly, this association was not seen for superficial $E$. coli infections. The high relevance of $E$. coli for urinary tract infections [30] (which were classed in the superficial infection group in this study) and their specific complications is a likely reason for this. Pathogenic factors associated with uropathogenic E. coli comprise fimbriae, curli, pili, capsules, iron scavenger receptors, flagella, toxins, and lipopolysaccharides $[25,26,30]$. In contrast, superficial E. coli infections were associated with a decrease of duration of hospital stay and the likelihood of infectious disease as the main diagnosis compared with systemic infections. Also in contrast, the prominence of $S$. aureus-associated skin and soft-tissue infections [50] allowed an association of superficial S. aureus infections and infectious disease as main diagnosis. Clumping factor B, Panton-Valentine leukocidin, and bi-component pore-forming toxins are prominent virulence factors that have been associated with skin and soft tissue infections due to S. aureus [51-54]. Admittedly, molecular screening for virulence factors was beyond the scope of this study.

Antibiotic drug resistance was only weakly associated with disease severity as measured by the chosen outcome parameters. Potential resistance factors associated with the observed disease severity-associated resistance patterns comprise ribosomal methylases or efflux pumps causing clindamycin resistance [55], tetracycline resistance genes of the tet gene family associated with tetracycline resistance [56], or fusidic acid resistance genes of the fus gen family associated with fusidic acid resistance [57] in $S$. aureus, as well as beta-lactamases associated with 3rd generation cephalosporin resistance in $E$. coli [55]. In all observed cases, higher levels of resistance were associated with increased disease severity. Accordingly, this hypothesis-forming assessment provided no indications supporting the trade-off theory of fitness cost and antibiotic resistance, as suggested in the literature [44, 45]. Admittedly, the study was only an explorative assessment, and pathogenic factors of the isolates were not assessed, an undeniable limitation of the study. Further, applied interpretation standards of resistance testing have been changed in the course of the 
study, which interferes with interpretability, although major discrepancies are nevertheless unlikely.

The study has a number of further limitations. Since the study is a retrospective exploration, conclusions regarding associative relationships can only provide hypotheses. In addition, especially in the global model of both groups with either $S$. aureus or E. coli and in the local model for patients with $S$. aureus, the models account for only a small part of the total dispersion, so it must be assumed that a considerable amount of information that could have been explanatory was not collected. However, the hypotheses derived from this study can be used to guide future prospective studies.

\section{Conclusion}

In conclusion, the study did not show specific association of disease severity, as defined by the endpoint parameters with the isolation of either $S$. aureus or E. coli, while, as expected, superficial infections were generally associated with milder diseases in comparison to systemic infections, as suggested by the outcome parameters. Interestingly, a reduced risk of death was shown for superficial infections due to $S$. aureus but not for those due to E. coli, in comparison to systemic infections. As far as can be determined despite the limitations of the study, resistance was associated with increased disease severity as defined by the endpoint parameters, so the phenomenon of trade-off between resistance and pathogenicity was not supported.

\section{Funding Sources}

No financial support was received for this study.

\section{Authors' Contributions}

$\mathrm{JU}, \mathrm{SB}$, and UJ conducted the collection and assessments of the data. AP and PW performed the laboratory assessments. $\mathrm{AH}$ and NGS were in charge of the statistical assessment. AH, AP, HF, and PW planned the design of the retrospective study. All authors jointly wrote and optimized the manuscript.

\section{Conflict of Interest}

Nothing to declare.

\section{References}

1. Armed Forces Health Surveillance Center (AFHSC). Septicemia diagnosed during hospitalizations, active component service members, U.S. Armed Forces, 2000-2012. MSMR. 2013;20:10-6.

2. Sherwood J, Park M, Robben P, Whitman T, Ellis MW. USA300 methicillin-resistant Staphylococcus aureus emerging as a cause of bloodstream infections at military medical centers. Infect Control Hosp Epidemiol. 2013;34:393-99.

3. Landrum ML, Neumann C, Cook C, Chukwuma U, Ellis MW, Hospenthal DR, et al. Epidemiology of Staphylococcus aureus blood and skin and soft tissue infections in the US military health system, 2005-2010. JAMA. 2012;308:50-9.

4. Ressner RA, Murray CK, Griffith ME, Rasnake MS, Hospenthal DR, Wolf SE. Outcomes of bacteremia in burn patients involved in combat operations overseas. J Am Coll Surg. 2008;206:439-44.

5. Weintrob AC, Murray CK, Xu J, Krauss M, Bradley W, Warkentien TE, et al. Early Infections Complicating the Care of Combat Casualties from Iraq and Afghanistan. Surg Infect. 2018;19:286-97.

6. Campbell WR, Li P, Whitman TJ, Blyth DM, Schnaubelt ER, Mende $\mathrm{K}$, et al. Multi-Drug-Resistant Gram-Negative Infections in DeploymentRelated Trauma Patients. Surg Infect. 2017;18:357-67.

7. Mende K, Beckius ML, Zera WC, Onmus-Leone F, Murray CK, Tribble DR. Low Prevalence of carbapenem-resistant Enterobacteriaceae among wounded military personnel. US Army Med Dep J. 2017;2-17:12-7.

8. Mende K, Beckius ML, Zera WC, Yu X, Cheatle KA, Aggarwal D, et al. Phenotypic and genotypic changes over time and across facilities of serial colonizing and infecting Escherichia coli isolates recovered from injured service members. J Clin Microbiol. 2014;52:3869-77.

9. Fisher A, Webber BJ, Pawlak MT, Johnston L, Tchandja JB, Yun H Epidemiology, microbiology, and antibiotic susceptibility patterns of skin and soft tissue infections, Joint Base San Antonio-Lackland, Texas, 2012-2014. MSMR. 2015;22:2-6.

10. Lamb L, Morgan M. Skin and soft tissue infections in the military. J R Army Med Corps. 2013;159:215-23.

11. Geerdes HF, Ziegler D, Lode H, Hund M, Loehr A, Fangmann W, Wagner J. Septicemia in 980 patients at a university hospital in Berlin: prospective studies during 4 selected years between 1979 and 1989. Clin Infect Dis. 1992;15:991-1002.

12. Rosenthal EJ. Septicemia causative organisms 1983-1985. The results of a multicenter study. Dtsch Med Wochenschr. 1986;111:1874-80.

13. Rosenthal EJ. Epidemiology of septicaemia pathogens. Dtsch Med Wochenschr. 2002; 127:2435-40.

14. Rosenthal EJ. The epidemiology of septicemia causative agents. A blood culture study of the Paul Ehrlich Society for Chemotherapy e. V. Dtsch Med Wochenschr. 1993;118:1269-75.

15. Schaberg DR, Culver DH, Gaynes RP. Major trends in the microbial etiology of nosocomial infection. Am J Med. 1991;91(3B):72S-5S.

16. Wisplinghoff H, Bischoff T, Tallent SM, Seifert H, Wenzel RP, Edmond MB. Nosocomial bloodstream infections in US hospitals: analysis of 24,179 cases from a prospective nationwide surveillance study. Clin Infect Dis. 2004:39:309-17.

17. Yaw LK, Robinson JO, Ho KM. A comparison of long-term outcomes after meticillin-resistant and meticillin-sensitive Staphylococcus aureus bacteraemia: an observational cohort study. Lancet Infect Dis. 2014;14:96775 .

18. Bhattacharya A, Nsonwu O, Johnson AP, Hope R. Estimating the incidence and 30-day all-cause mortality rate of Escherichia coli bacteraemia in England by 2020/21. J Hosp Infect. 2018;98:228-31.

19. Rhodes A, Evans LE, Alhazzani W, Levy MM, Antonelli M, Ferrer R, et al. Surviving Sepsis Campaign: International Guidelines for Management of Sepsis and Septic Shock: 2016. Intensive Care Med. 2017;43:304-77.

20. Jung N, Rieg S. Essentials in the management of $S$. aureus bloodstream infection. Infection. 2018;46:441-2.

21. Azzopardi EA, Azzopardi E, Camilleri L, Villapalos J, Boyce DE, Dziewulski P, et al. Gram negative wound infection in hospitalised adult burn patients-systematic review and metanalysis. PLoS One. 2014;9:e95042.

22. Bessa LJ, Fazii P, Di Giulio M, Cellini L. Bacterial isolates from infected wounds and their antibiotic susceptibility pattern: some remarks about wound infection. Int Wound J. 2015;12:47-52.

23. Serra R, Grande R, Butrico L, Rossi A, Settimio UF, Caroleo B, et al Chronic wound infections: the role of Pseudomonas aeruginosa and Staphylococcus aureus. Expert Rev Anti Infect Ther. 2015;13:605-13.

24. Karakonstantis S, Kalemaki D. Evaluation and management of Staphylococcus aureus bacteriuria: an updated review. Infection. 2018;46:293301 .

25. Terlizzi ME, Gribaudo G, Maffei ME. UroPathogenic Escherichia coli (UPEC) Infections: Virulence Factors, Bladder Responses, Antibiotic, and Non-antibiotic Antimicrobial Strategies. Front Microbiol. 2017;8:566.

26. Lüthje P, Brauner A. Virulence factors of uropathogenic E. coli and their interaction with the host. Adv Microb Physiol. 2014;65:337-72.

27. Otto M. Staphylococcus aureus toxins. Curr Opin Microbiol. 2014;17:32-7.

28. Tapader R, Basu S, Pal A. A regulatory trade-off as a source of strain variation in the species Escherichia coli. J Bacteriol. 2004:86:5614-20.

29. Sarowska J, Futoma-Koloch B, Jama-Kmiecik A, Frej-Madrzak M, Ksiazczyk M, Bugla-Ploskonska G, et al. Virulence factors, prevalence and potential transmission of extraintestinal pathogenic Escherichia coli isolated from different sources: recent reports. Gut Pathog. 2019;11:10.

30. Asadi Karam MR, Habibi M, Bouzari S. Urinary tract infection: Pathogenicity, antibiotic resistance and development of effective vaccines against Uropathogenic Escherichia coli. Mol Immunol. 2019;108:56-67.

31. Johnson JR, Russo TA. Molecular Epidemiology of Extraintestinal Pathogenic Escherichia coli. EcoSal Plus. 2018;8:1.

32. Mainil J. Escherichia coli virulence factors. Vet Immunol Immunopathol. 2013;152:2-12.

33. Tam K, Torres VJ. Staphylococcus aureus Secreted Toxins and Extracellular Enzymes. Microbiol Spectr. 2019;7:2.

34. Singh V, Phukan UJ. Interaction of host and Staphylococcus aureus protease-system regulates virulence and pathogenicity. Med Microbiol Immunol. 2018, [Epub ahead of print]:doi: 10.1007/s00430-018-0573-y.

35. Oliveira D, Borges A, Simões M. Staphylococcus aureus Toxins and Their Molecular Activity in Infectious Diseases. Toxins, 2018;10:6.

36. Hecker M, Mäder U, Völker U. From the genome sequence via the proteome to cell physiology - Pathoproteomics and pathophysiology of Staphylococcus aureus. Int J Med Microbiol. 2018;308:545-57.

37. Dayan GH, Mohamed N, Scully IL, Cooper D, Begier E, Eiden J, et al. Staphylococcus aureus: the current state of disease, pathophysiology and strategies for prevention. Expert Rev Vaccines. 2016;15:1373-92.

38. Thomer L, Schneewind O, Missiakas D. Pathogenesis of Staphylococcus aureus Bloodstream Infections. Annu Rev Pathol. 2016;11:343-64

39. Becker RE, Bubeck Wardenburg J. Staphylococcus aureus and the skin: a longstanding and complex interaction. Skinmed. 2015;13:111-9.

40. Tong SY, Davis JS, Eichenberger E, Holland TL, Fowler VG Jr. Staphylococcus aureus infections: epidemiology, pathophysiology, clinical manifestations, and management. Clin Microbiol Rev. 2015;28:603-61.

41. Powers ME, Bubeck Wardenburg J. Igniting the fire: Staphylococcus aureus virulence factors in the pathogenesis of sepsis. PLoS Pathog. 2014;10: e1003871.

42. Frickmann H, Podbielski A, Kreikemeyer B. Resistant Gram-Negative Bacteria and Diagnostic Point-of-Care Options for the Field Setting during Military Operations. Biomed Res Int. 2018;2018:9395420. 
43. Frickmann H. Impact of MRSA on the Military Medical Service and Diagnostic Point-of-Care Options for the Field Setting. Eur J Microbiol Immunol. 2018;8:31-3.

44. King T, Ishihama A, Kori A, Ferenci T. A regulatory trade-off as a source of strain variation in the species Escherichia coli. J Bacteriol. 2004; $186: 5614-20$

45. Ferenci $\mathrm{T}$. What is driving the acquisition of mutS and rpoS polymorphisms in Escherichia coli? Trends Microbiol. 2003;11:457-61.

46. Jo SJ, Park KG, Han K, Park DJ, Park YJ (2016) Direct identification and antimicrobial susceptibility testing of bacteria from positive blood culture bottles by Matrix-Assisted Laser Desorption/Ionization Time-of-Flight Mass Spectrometry and the Vitek 2 system. Ann Lab Med. 2016:36:117-23.

47. Angeletti S. Matrix assisted laser desorption time of flight mass spectrometry (MALDI-TOF-MS) in clinical microbiology. J Microbiol Meth 2017;138:20-9.

48. Kassim A, Pflüger V, Preemji Z, Daubenberger C, Revathi G. Comparison of biomarker based Matrix Assisted Laser Desorption IonizationTime od Flight Mass Spectrometry (MALDI-TOF-MS) and conventional methods in the identification of clinically relevant bacteria and yeast. BMC Microbiology. 2017; 17:128

49. Trevisoli LE, Bail L, Rodrigues LS, Conte D, Palmeiro JK, Dalla-Costa LM. Matrix-assisted laser desorption ionization-time of flight: a promising alternative method of identifying the major coagulase-negative Staphylococci species. Rev Soc Bras Med Trop. 2018;51:85-7.

50. Poulakou G, Lagou S, Tsiodras S. What's new in the epidemiology of skin and soft tissue infections in 2018? Curr Opin Infect Dis. 2019;32:77-86.
51. Lacey KA, Mulcahy ME, Towell AM, Geoghegan JA, McLoughlin RM. Clumping factor B is an important virulence factor during Staphylococcus aureus skin infection and a promising vaccine target. PLoS Pathog 2019;15: e1007713.

52. Klein S, Menz MD, Zanger P, Heeg K, Nurjadi D. Increase in the prevalence of Panton-Valentine leukocidin and clonal shift in community-onset methicillin-resistant Staphylococcus aureus causing skin and soft-tissue infections in the Rhine-Neckar Region, Germany, 2012-2016. Int J Antimicrob Agents. 2019;53:261-7.

53. Perumal N, Dass BS, Mani S, Krishnan P. Prevalence of bi-component pore-forming toxin genotypes of Staphylococcus aureus causing skin and soft tissue infections. Indian J Med Microbiol. 2017:35:146-7.

54. Jahamy H, Ganga R, Al Raiy B, Shemes S, Nagappan V, Sharma M, Riederer K, Khatib R. Staphylococcus aureus skin/soft-tissue infections: the impact of SCCmec type and Panton-Valentine leukocidin. Scand J Infect Dis. 2008;40(8):601-6.

55. Leclercq R, Cantón R, Brown DF, Giske CG, Heisig P, MacGowan AP, Mouton JW, Nordmann P, Rodloff AC, Rossolini GM, Soussy CJ, Steinbakk $\mathrm{M}$, Winstanley TG, Kahlmeter G. EUCAST expert rules in antimicrobial susceptibility testing. Clin Microbiol Infect. 2013;19:141-60.

56. Schwarz S, Cardoso M, Wegener HC. Nucleotide sequence and phylogeny of the $\operatorname{tet}(L)$ tetracycline resistance determinant encoded by plasmid pSTE1 from Staphylococcus hyicus. Antimicrob Agents Chemother. 1992;36:580-8.

57. Chen HJ, Hung WC, Lin YT, Tsai JC, Chiu HC, Hsueh PR, Teng LJ. A novel fusidic acid resistance determinant, fus $F$, in Staphylococcus cohnii. J Antimicrob Chemother. 2015;70:416-9. 